\title{
PRÁCTICAS DE APROPIACIÓN TERRITORIAL: \\ EL CASO DE LA ACUICULTURA EN EL MAR INTERIOR DE CHILOÉ*1
}

\author{
TERRITORIAL APPROPRIATION PRACTICES: \\ THE CASE OF AQUACULTURE IN THE INLAND SEA OF CHILOÉ
}

\author{
Ítalo Carrera Arenas ${ }^{2}$ \\ italocarrera@hotmail.com \\ Universidad de Los Lagos \\ Osorno, Chile \\ DOI: https://doi.org/10.32735/S2735-61752019000116141
}

\section{RESUMEN}

En el contexto del desarrollo de la tesis de doctorado "Estudio en torno a apropiaciones territoriales, transformaciones socio-ecológicas y discursos científicos en el maritorio interior de Chiloé" de la Universidad de Los Lagos, el presente texto tiene por objeto describir y analizar -a partir de una concepción histórico-antropológica- la práctica de apropiación territorial de la acuicultura, que ha ido reconfigurando aspectos sociales y culturales del borde costero Chilote a través de una relación ambiental conflictiva para/con la naturaleza.

Palabras claves: acuicultura, maritorio interior de Chiloé, apropiación territorial, conflicto socioambiental.

\begin{abstract}
In the context of the development of the doctoral thesis "Study regarding to the territorial appropriation, socio-ecological transformations and scientific discourse in the Inland Sea of Chiloé" from Los Lagos University, the following text is intended to describe and analize - from an historical and anthropological conception - the practice of the territorial appropriation of aquaculture. That have reconfigured social and cultural aspects in the coastline through a troubled environmental relation with nature.
\end{abstract}

Key words: Aquaculture, inland sea of Chiloé, territorial appropriation.

\footnotetext{
*Artículo recibido el 8 de septiembre de 2017; aceptado el 10 de noviembre de 2017.

${ }^{1}$ Esta investigación está, en parte, al resultado del proyecto doctoral en curso.

2 Doctor (c) en Ciencias Sociales en Estudios Terroriales, Centro de Estudios del Desarrollo Regional y Políticas Públicas CEDER, por la Universidad de Los Lagos.
} 


\section{Introducción}

El actual conflicto socioecológico en el maritorio interior de Chiloé y en el litoral de la Región de Los Lagos, convoca a las ciencias sociales al desarrollo de investigaciones interdisciplinares y/o multidisciplinarias que contribuyan a la comprensión de -y en torno a- la naturaleza de dichos fenómenos, los cuales se expresan a través de la relación dialógica entre el cultura (occidental) y naturaleza. El actual proceso socioecológico de Chiloé, como producto de la apropiación territorial e intervención tecno-científica de la salmonicultura, nos indica que la trasformación sin mesura de la naturaleza por los sistemas culturales (y modelos de organización social) modernos ha llegado a un punto de incertidumbre respecto de sus efectos, donde la naturaleza modificada-intervenida (ambiente transformado) revierte dicho daño trayendo como consecuencia la destrucción (o riesgo de ello) de los sistemas culturales y el conjunto de prácticas que lo determinan, al respecto, la literatura ya nos señalaba que "la sociedad se pone en peligro a sí misma, en la medida en que produce efectos en el medio ambiente" (Luhmann, 2012 , p. 82). Dichas transformaciones se desarrollan con más fuerza desde la década del 90 " a partir de prácticas de apropiación -que ancladas en los discursos científicos pro desarrollo capitalista- se expresan a través de figuras económicas como la salmonicultura, pesca de arrastre y llegada de servicios como agua potable y luz eléctrica, entre otros, generando procesos acelerados de intervención y cambio de los entornos locales, modificando prácticas, discursos locales, identidades e imaginarios. En ese contexto, los conflictos socioecológicos serían el resultado de las formas de apropiación del territorio (prácticas y discursos), y su comprensión exige un abordaje integral complementando dimensiones históricas, antropológicas y biológicas.

En el contexto de significativas y aceleradas transformaciones en los territorios locales, que derivan del proceso de modernización que han vivido los países de Latinoamérica en las últimas décadas y con ello la introducción de distintos modelos de producción económica y configuraciones tecno-industriales de apropiación de la naturaleza y del territorio, podemos señalar -y compartir la idea de- que los conflictos socioecológicos y crisis socioambientales tienen origen en la racionalidad y por ende tanto en nuestros conocimientos sancionados como civilización como en nuestros discursos anclados en la ciencia, "esta encrucijada civilizatoria es ante todo una crisis de la racionalidad de la modernidad y remite a un problema del conocimiento" (Leff, 2006, p. 192).

Conocer los tipos de apropiaciones territoriales en el mar interior de Chiloé es una primera aproximación a la comprensión de los fenómenos socioecológicos que allí acontecen.

En el presente texto, junto con describir y analizar desde una concepción históricoantropológica- la práctica de apropiación territorial de la acuicultura y sus efectos sociales y culturales del borde costero Chilote, intentaremos permitirnos desarrollar una breve contextualización histórica de Chiloé para luego contribuir con una sistematización y recuperación de la historia de la acuicultura, a partir de ahí responder ¿En qué periodos y etapas evoluciona la acuicultura (salmoncultura), ¿Qué actores están involucrados?, ¿a través de qué procesos se desarrolla?, ¿Cómo marcaron la transición hacia una reapropiación y redefinición del territorio local? Para ello, recurriremos a los discursos históricos, antropológicos y biológicos asociados.

\section{Descripción territorio de Chiloé}

El Archipiélago de Chiloé, lugar en el cual existe la segunda Isla más grande de Sudamérica y que fuera tierra ancestral de los pueblos Huilliche, Chono y Cunco, es un territorio de particular interés para las investigaciones de ciencias sociales y de los discursos que se han edificado en 
torno a él, dado que durante las últimas tres décadas -en dicho lugar- se han desarrollado -de forma acelerada- significativas transformaciones ambientales, culturales, sociales, económicas y geopolíticas, sobre todo en torno al mar interior de Chiloé cuya constitución está dada por los Golfos de Ancud, del Corcovado y del seno del Reloncaví. Este territorio, que ha vivido continuas transformaciones desde hace siglos y cuya referencia histórica -solo a modo de contextualización- se inicia desde el año 1567 a través de la conquista española, queda ubicado en la Región de Los Lagos, Chile, cuyas coordenadas son $42^{\circ} 40^{\prime \prime} 36^{\prime \prime} \mathrm{S} 73^{\circ} 59^{\prime} 36^{\prime \prime} \mathrm{O}$, y a más de mil kilómetros de distancia de la capital Santiago de Chile. Su superficie ha sido calculada en $8.394 \mathrm{~km}^{2}$ con una longitud de $180 \mathrm{~km}$ y una población aproximada de 180 mil personas. Su mayor punto de conectividad se desarrolla a través del canal de Chacao en el sector de Pargua vía transbordadores comerciales -a un poco más de 70 kilómetros de la capital regional Puerto Montt. Este espacio se encontraba habitado desde tiempos inmemoriales y hasta el año 1567, mayoritariamente por bandas nómades dedicadas a la caza, pesca y recolección costera, esto último testificado por numerosos conchales. Algunos de los grupos indígenas que se localizaron en la isla fueron los Chono, Huilliche y Cunco. En el caso de los Chono, estos fueron invadidos y asimilados, en parte, por los huilliche provenientes del territorio continental al norte del Canal de Chacao, los que posteriormente se autodominaron Cunco. Conforme a su patrón económico y nivel de estructura social, los Cunco han sido calificados como pescadores y horticultores agrupados en tribus sedentarias.

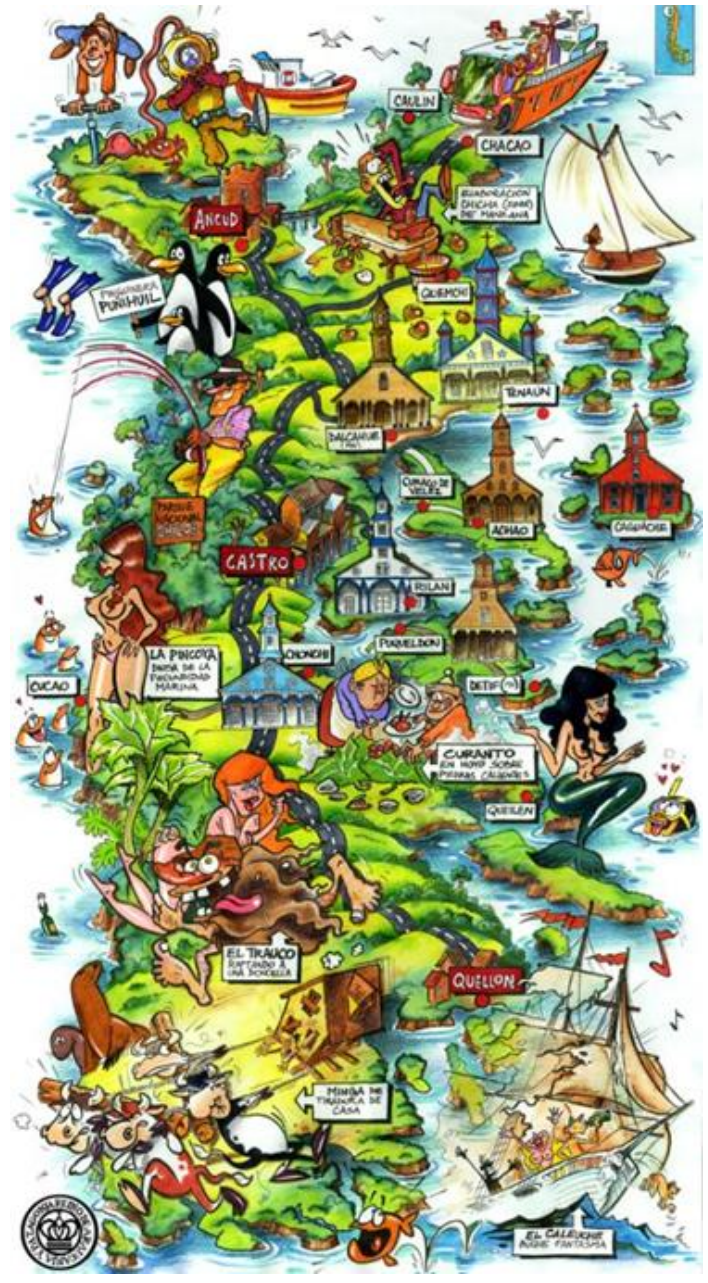

Figura 1. Chiloé mítico-mágico. Fuente: https://www.xn--turismochilo-meb.com/

Las actividades agrícolas principales ejercidas por ellos, consistían en cultivos de papa, el fríjol y la quínoa (otros productos como semilla de trigo, cebada, avena, linaza, ajos, cebollas, arvejas, fueron introducidos por los españoles).

Por otro lado, para comunicarse entre las diversas islas y para sus viajes al continente, se servían de pequeñas embarcaciones (bongos y dalcas). Estos antecedentes nos señalan una ocupación costera del archipiélago, con pocos asentamientos permanentes que posibilitaran una estructura social, económica y política más compleja. El carácter costero que desde un principio tuvieron los asentamientos españoles, se originaron producto de un proceso 
colonizador concebido a partir de una base urbana; el traslado hacia las zonas interiores significaba no sólo dispersión de la población española, sino también la ruralización de ella. En este marco conceptual, se construirá la identidad de cada poblado apropiado por los españoles, su densidad poblacional y su relación con el medio. Los primeros reconocimientos por mar de la zona insular, fueron realizados por Alonso de Camargo en 1540 y, 5 años más tarde, en 1545, por Francisco de Ulloa que en su expedición al Estrecho de Magallanes, reconoció la costa de la isla y archipiélagos vecinos, siendo catalogado como su descubridor y primer explorador. En 1597, Martín Ruiz de Gamboa (fundador de la ciudad de Castro) anexó definitivamente el archipiélago a la Corona Española, Ilamándolo Nueva Galicia.

La anexión del nuevo territorio, fue relativamente tranquila y sin situaciones bélicas notables, debido a la escasa oposición presentada por los indígenas. Administrativamente el Archipiélago de Chiloé, estuvo desde su descubrimiento bajo la jurisdicción del Reino de Chile. Las dificultades en las comunicaciones y la dependencia establecida a través del Real Situado, generaron su tardía incorporación al Virreinato del Perú (1767). Desde su conquista hasta 1598, el archipiélago se comportó como Frontera Abierta, esto es, como un espacio en proceso de colonización franco por parte de españoles, los cuales impulsaron nuevas conquistas hacia los territorios australes, lo que afianzaría la expansión y conquista del Estrecho de Magallanes.

Desde su conquista en 1567, Chiloé presenta un poblamiento inestable; incrementándose desde que se transforma en Frontera Cerrada, luego de la Rebelión General India de 1598 1604. Su historia escrita más conocida por los hoy pobladores del territorio se remonta a 1768 cuando el gobernador español don Carlos de Berenguer funda la ciudad de Ancud la cual cumplió en sus comienzos funciones de fortaleza para resguardar el tráfico por el Cabo de Hornos, esto dio a Ancud un prestigio y preponderancia que la convirtieron en la capital política y militar de este territorio en ese entonces.

El siglo XVII, comienza con un cambio que produce un quiebre en la fisonomía social, cultural, político y económica, esto es el paso a una Frontera Cerrada que significa la ruptura del vínculo con Chile, en el sentido que no sólo recibía el flujo de nuevos inmigrantes españoles, sino que también se impusieron restricciones a la salida de la población asentada en Chiloé. La característica del siglo XVII es la tendencia al despoblamiento total de españoles e indios, situación que dejaba a Chiloé en la más completa incomunicación, sobreviniendo la extrema pobreza y la exposición constante de los asentamientos a ataques extranjeros, tanto de corsarios como piratas interesados en el territorio. La idea de conservar territorios estratégicos por parte de la Corona española, dará pie a nuevos criterios de fundación, que en la planificación de las futuras ciudades se manifestará desde un principio por su marcado origen defensivo, iniciándose en el siglo XVIII el período de las fortificaciones.

En el contexto cultural antes planteado, se enmarca la fundación de Ancud. En el contexto general, Chiloé distaba mucho de ser una zona de tránsito, como son normalmente los territorios continentales, ni tampoco una región terminal donde concluyen las oleadas migratorias, por el contrario, se constituyó en un enclave y frontera cerrada, territorio rodeado de pueblos cuya vida se desenvolvía hacia el interior, en un proceso de continuos intercambios con la población indígena. Este siglo es de gran importancia, ya que marca otros criterios para el surgimiento de las ciudades, donde el paisaje y las condiciones naturales del lugar elegido eran determinantes. No eran ciudades de un sólo propósito, debían ser más integrales (defensivas y comerciales a la vez). De esta forma, Chiloé se constituyó, por sus cualidades naturales, en un importante territorio y circuito estratégico de defensa.

El movimiento revolucionario que venía generándose en Chile, cuya primera manifestación práctica fue el 18 de septiembre de 1810, pasó inadvertido en Chiloé por largo tiempo, ya que 
este territorio estaba padeciendo en grado superior del mismo problema de siglos anteriores, la incomunicación.

Esta situación dejó al archipiélago con una economía altamente precarizada, el comercio limitó sus transacciones, decayendo a un extremo lamentable por la escasez de naves con que transportar las maderas y productos agrícolas. Bajo ese panorama, llegó el archipiélago a su independencia. Es así, que en 1826 la expedición comandada por el propio Director Supremo Ramón Freire, se apoderó de los fuertes Ahui, Punta Corona y de las posiciones artilladas Chaicura, Balcacura y Puquillihue, hasta conquistar la plaza de San Carlos y provocar la derrota del ejército realista al mando del brigadier español Antonio de Quintanilla. El 19 de enero se ratificaba la rendición junto al río San Antonio, y el día 22 se proclamó la independencia del archipiélago y su incorporación al territorio nacional.

Según la ley promulgada el 30 de agosto de 1826, Chiloé formó una de las 8 provincias en que fue dividido el territorio nacional, involucrando los departamentos de Ancud (capital), Calbuco, Carelmapu, Castro, Chacao, Chonchi, Dalcahue, Lemuy, Quenac y Quinchao. Por Decreto Supremo del 28 de febrero de 1855, los 10 departamentos anteriores quedaron reducidos a 4: Ancud (capital), Castro, Quinchao y Carelmapu. Posterior a 1960, con la construcción de la Carretera Panamericana que unía los principales puntos del país, la ciudad de Puerto Montt se constituye como un centro económico y comercial de relevancia nacional, rol que se conserva hasta nuestros días.

\section{Dinámicas de apropiación tecno-científicas; El caso de la acuicultura-salmonicultura}

Existen tres periodos importantes ${ }^{3}$ en el proceso histórico/antropológico de la apropiación territorial tecno-científica a través del cual se desarrollan transformaciones territoriales significativas, en Chiloé:

\footnotetext{
${ }^{3}$ Para comprender el caso de la acuicultura, como expresión de un sistema-tipo de apropiación tecnocientífica anclada en el discurso antropocentrista e imaginario capitalista, utilizaremos como carta de navegación - en el contexto de sus datos- el texto "Historia de la acuicultura", impreso el 2003 y que registra detalladamente aspectos fundamentales para comprender décadas de transformaciones subyacentes a la acuicultura. A pesar de no tener un autor concreto, identificaremos las citas como: Martínez, S. (2003). Historia de la acuicultura. TechnoPress S.A. Editorial de AquaNoticias. $1^{\circ}$ ED. Directorio de Acuicultura y Pesca de Chile SalmónChile, Santiago, Chile./ Sergio Martínez es Ingeniero Eléctrico y actor importante de los primeros procesos de instalación de la acuicultura, quien amablemente nos facilitó uno de los escasos ejemplares existentes de dicha edición. Fue el director del proyecto de investigación.
} 


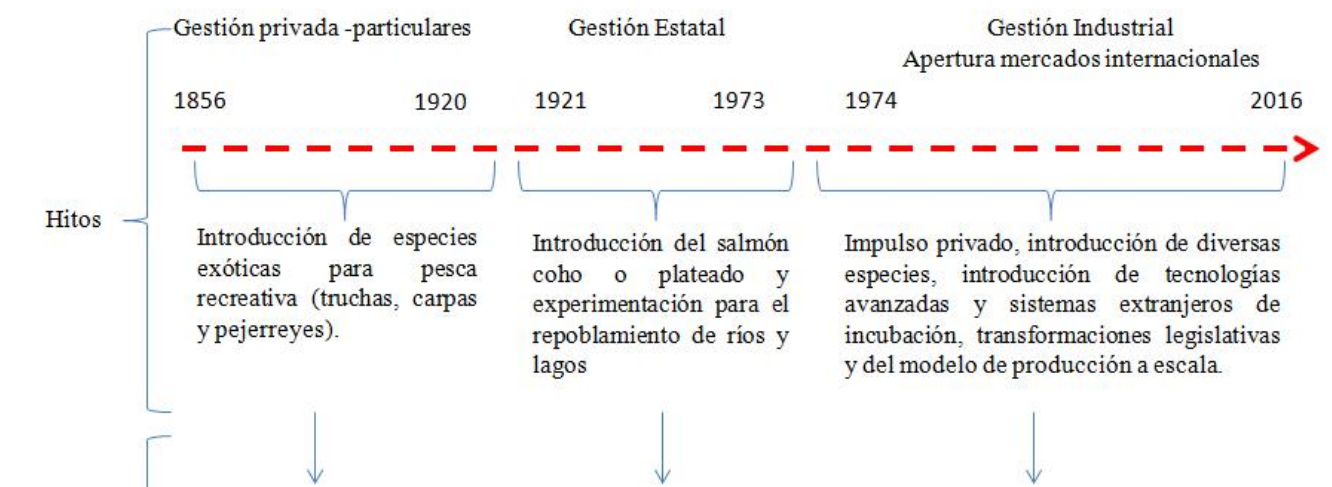

División de Pesca y Casa, $\begin{array}{ll}\text { Tomas Urmenta } & \text { SAG, del Ministerio de } \\ \text { Isidora Goyenechea } & \text { Agricultura, FAO, IFOP }\end{array}$ $\begin{array}{ll}\text { Tomas Urmenta } & \text { SAG, del Ministerio } \\ \text { Isidora Goyenechea } & \text { Agricultura, FAO, IFOP }\end{array}$

CORFO, Sernapesca, Sociedad de Pesquerías, Salmones Llanquihue, Unión Carbide, Instituto Tecnológico de Puerto Montt, Domsea Pesquera Chile, entre otros.
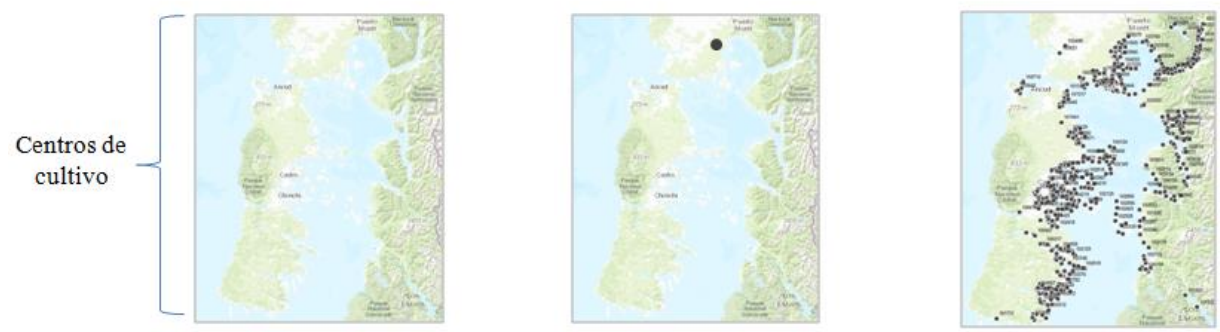

Figura 2. Periodos de la acuicultura/salmonicultura en Chile y Chiloé. Fuente: elaboración del autor sobre la base de lo dicho en la nota al pie 3.

La acuicultura, descrita de una manera sencilla, es la técnica que permite aumentar la producción de animales y plantas acuáticas para consumo humano y su comercialización por medio de cierto control de los organismos y de su medio ambiente. En el caso de la salmonicultura, el método más sofisticado consiste en la eclosión de huevos, la cría de juveniles en estanques $u$ otros corrales hasta que alcanzan el tamaño comercial, y el mantenimiento de los pies de cría. El salmonicultor - a través de la técnica- logra un completo control sobre el ciclo de vida del animal, desde las ovas en los lagos, hasta los alevines en los centros de cultivo de mar, para ello, deberá considerar el proceso biológico y comercial -de principio a fin- como un sistema racional.

"La industria de la salmonicultura, se define a sí misma como un área productiva joven, de 35 años, como una mixtura entre el espíritu emprendedor chileno y, de acuerdo al actual director de Salmon Chile" (Felipe Sandoval, 2016), ${ }^{4}$ nace a partir de una necesidad emergente en Japón, que era la necesidad de mayor cantidad de proteínas en su mercado pesquero. Actualmente cuenta con más de 700 centros productivos, 73.520 empleos, de los cuales 30.387 son directos, 43.133 son indirectos, $30 \%$ son mujeres y otro $30 \%$ mano de obra juvenil. Esto

\footnotetext{
${ }^{4}$ Exposición Seminario Crisis en el Sur, desarrollado por la Universidad Austral el día viernes 8 de julio de 2016.
} 
significa el $61 \%$ de personal a nivel mundial dedicado a la industria del salmón -industria que requiere más de 70 mil hectáreas agrícolas para la producción de trigo, lupino y raps canola como insumos para la producción de peces- radica en Chile.

La enorme faja costera del sur austral cuyas aguas de excelentes temperaturas y numerosas islas, no han estados ajenas a los ojos de la economía neoliberal y del imaginario capitalista tecno-científico, ello ha permitido que la acuicultura - fundamentalmente la salmonicultura- sea hoy una de las actividades económicas qué más ha dado que hablar en los últimos tiempos, fundamentalmente por su potencial asociación a una de las peores crisis socioecológicas que haya vivido el litoral del mar interior de Chiloé y las costas del pacífico durante el 2016. En términos económicos, la apropiación del mar interior de Chiloé como de todo el sur austral, es concebida como un éxito, dado el gran éxito financiero independiente de las secuelas ambientales. Este éxito está asociado al llamado "cinturón del salmón", una extensión de alrededor de 1700 kilómetros entre Puerto Montt y Punta Arenas con condiciones óptimas para cultivo.

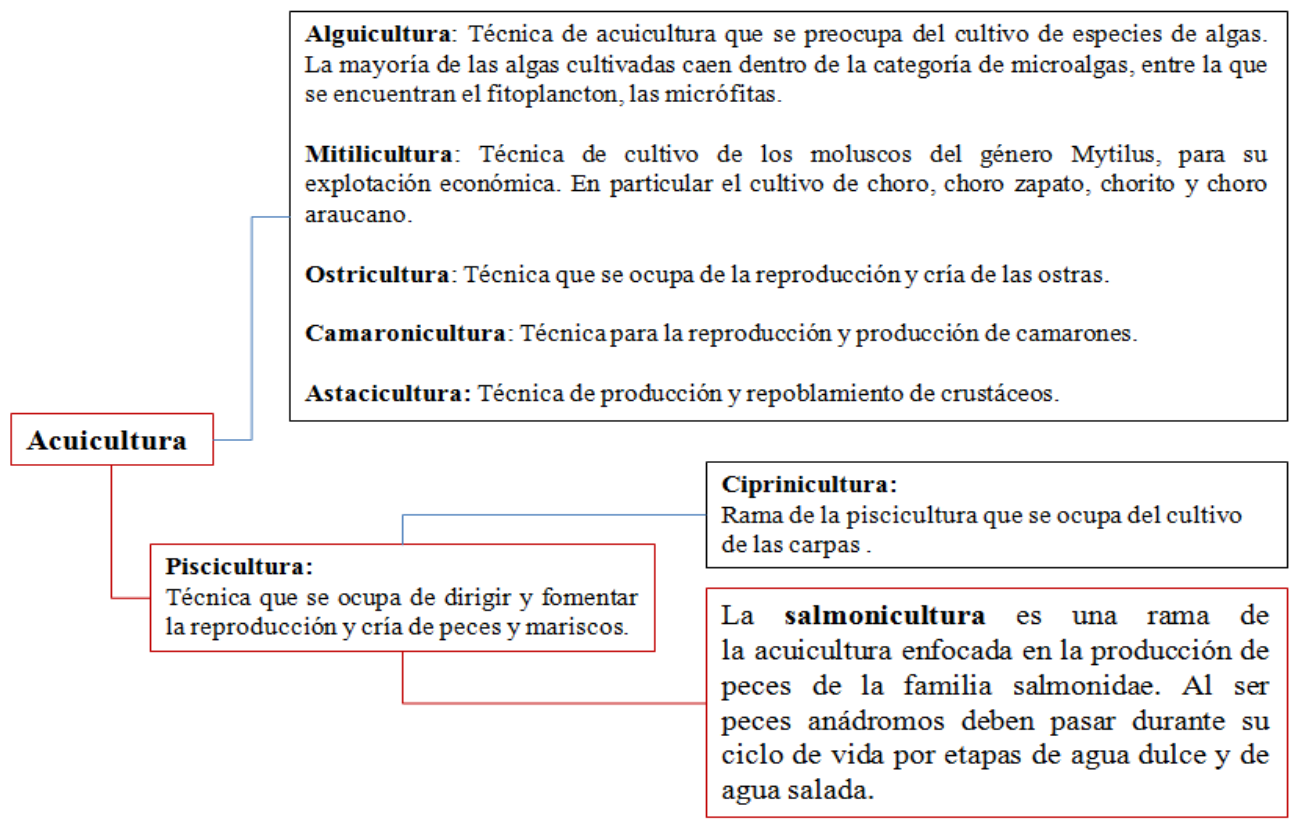

Figura 3. Disciplinas de la acuicultura. Fuente: elaboración propia del autor.

En el desarrollo de la acuicultura en Chile, desde una perspectiva histórico/antropológica, se pueden distinguir tres periodos importantes. El primero se sitúa entre 1850 y 1920, cuando se inició en Chile la etapa de introducción de especies acuícolas exóticas, como truchas, carpas y pejerreyes, para potenciar la pesca recreativa. El segundo período, entre 1921 y 1973, se caracterizó por los esfuerzos orientados a la introducción del salmón coho o plateado (Oncorhynchus kisutch) y por establecer actividades económicas comerciales relacionadas con la acuicultura. En esto último el Instituto de Fomento Pesquero (IFOP) tuvo un rol destacado en cuanto a implantar en el país tecnologías extranjeras pioneras y necesarias para el cultivo de distintas especies acuícolas y traer a expertos internacionales para transmitir e instalar esos "conocimientos especializados". En el tercer periodo, que comienza en 1974 y se extiende hasta 
el día de hoy, la actividad a nivel comercial se ha consolidado mediante una política económica de impulso a la gestión privada y apertura de los mercados internacionales, principalmente a través de los tratados de libre comercio. El aporte estatal, si bien se ha reducido ostensiblemente desde la perspectiva del empresariado, con el tiempo se ha ido concentrando sólo en un apoyo indirecto a través de la apertura y entrega de fondos concursables para proyectos de investigación, desarrollo tecnológico y evaluaciones diagnósticas, entre otros. A lo largo del camino, cada especie de cultivo ha tenido su propia historia y, en éste contexto, la salmonicultura se erige como la actividad más importante. Se muestran aquí la subestructura de la acuicultura, y nos concentraremos en adelante en la salmonicultura.

\section{Etapa 1: Gestión privada - particulares}

A partir de 1850 comenzó el interés por introducir en el país el salmón, una especie cuyos principales representantes son los salmónidos (del género Salmo) que habitan las frías aguas del hemisferio norte. El gran atractivo que el salmón tiene para el humano, además de su carne, es su comportamiento migratorio, puesto que nace en los ríos, migra hacia al mar en la vida adulta y remonta luego de miles de kilómetros para desovar en el mismo río de origen. En 1868 se creó la primera piscicultura (centro de cultivo en agua dulce) para producir salmones en Troutdale, provincia de Cumberland, Inglaterra. En 1871 se construyó en Estados Unidos una piscicultura en Orlando, Maine, y al año siguiente se levantó en ese país una segunda planta a orillas del río McCloude, afluente del río Sacramento, en California, para cultivar salmón coho o del Pacífico. Las distintas variedades del salmón habían sido identificadas en el siglo XIX por el naturista alemán George Wilhem Stellar, profesor de la Academia de Ciencias de San Petersburgo en Rusia, y corresponden al salmón sockeye o rojo (Oncorhynchus nerka); rosado (Oncorhynchus gorbuscha); coho o plateado; chum o perro (Oncorhynchus keta); Chinook o rey (Oncorhynchus tshawytscha) y cereza (Oncorhynchus masau).

En 1876 el japonés Akekiyo Sekizama realizó la primera incubación artificial de salmones, a partir de 17 mil ovas (huevos), obtenidas de ejemplares capturados en el río Nakagawa, cerca de la ciudad de Mito. Hasta 1888 se realizaron experimentos de incubación artificial y liberaciones en distintos lugares de la isla Honshu sin lograr grandes progresos, pero el mismo año, otro japonés, Katusaka Itoh, desarrolló una nueva técnica de incubación y fundó la piscicultura estatal en Chitose, en la isla de Hokkaido, que es hoy la principal abastecedora de salmón en Japón.

Así, Estados Unidos e Inglaterra lideraron los esfuerzos por introducir el salmón coho en distintos países europeos y sudamericanos. La primera incorporación exitosa en el hemisferio sur la realizó Inglaterra al enviar ovas a sus colonias en Australia y Nueva Zelanda. En Chile, el interés por importar ovas de salmones y truchas desde el hemisferio norte se inició hacia 1885 para poblar ríos, lagos y mar con fines deportivos-recreativos.

Las primeras iniciativas fueron lideradas por particulares, como el naturista alemán Federico Albert, quién en 1856 introdujo en el país la carpa china (Ciprinus carpa), hoy distribuida en diversos lagos y cursos de agua a lo largo de todo el territorio. También fueron Tomás Urmeneta y a Isidora Goyenechea de Cousiño, quienes en 1875 y 1885 respectivamente, hicieron esfuerzos por potenciar la introducción de truchas en el territorio nacional. Les siguieron Julio Besnard, Mac Clure y Bavari. Si bien estos intentos no tuvieron éxito esperado, debido a la lentitud e inseguridad con que operaban los medios de transporte marítimos que trasladaban las ovas en tinajas de madera, sí sembraron la inquietud por cultivar salmones y contribuyeron a sentar las bases de las pisciculturas que funcionan hasta hoy y a formar generaciones de técnicos piscicultoras. 
A principios del siglo XX se generó el desarrollo de la industria de la salmonicultura en el hemisferio norte. Recién después de la segunda guerra mundial, al declinar la abundancia del salmón silvestre en el pacífico norte debido a la creciente actividad pesquera, Japón, la Unión Soviética, Estados Unidos y Canadá iniciaron el cultivo artificial del salmón a gran escala mediante un sistema acuático en cautiverio, en el cual los ejemplares juveniles se criaban a partir de ovas en sistemas cerrados en agua dulce, para ser liberados posteriormente en aguas marinas.

En 1905, el Estado chileno comenzó a manifestar interés por el tema. Gracias a su iniciativa llegó al país una pequeña porción de ovas de salmón del Atlántico (Salmo salar) y trucha arco iris o salmonídea (Oncorhynchus mykiss) -especie canadiense originaria de aguas dulces-, compradas en Hamburgo (Alemania) por Pedro Golusda y Rudolf Wilde. Debido a su avanzado estado de desarrollo, fueron desembarcadas en Buenos Aires, transportadas por tren a Mendoza y luego a "lomo de mula" a través de la cordillera de los Andes hasta llegar a la piscicultura de río Blanco, en la provincia de Los Andes, V Región. Construida en 1904. Allí se produjo la primera eclosión de salmónidos en el país y se dio inicio al cultivo confinado de truchas en estanque y su introducción en ríos y lagos. Entre 1905 y 1910 se efectuaron importaciones continuas de ovas y se sembraron los primeros alevines (peces en la etapa que comprende desde su nacimiento hasta el inicio de su adaptación al medio marino) en el río Aconcagua, en la zona central, y en Los ríos Maule, en la VII región; Toltén y Cautín, en la IX Región y Rahue, Maullín y Petrohué, en la Región de Los Lagos.

En 1914 se capturaron las primeras truchas adultas provenientes de las siembras en la piscicultura Lautaro, a orillas del río Cautín en la zona del mismo nombre. Ello aceleró la construcción de una segunda piscicultura a orillas de ese curso de agua con el propósito de introducir especies salmónidas en el centro y sur del país. Dos años más tarde desovó allí la primera camada de truchas arcoíris y salmón del Atlántico, transformándose esa segunda piscicultura en la cuna del desarrollo de la pesca deportiva en Chile, ya que muchas de sus ovas se trasladaron con ese objetivo a la zona de Magallanes, XII Región, donde esa actividad se sigue practicando exitosamente. De esta manera, hasta 1937 se lograron establecer importantes poblaciones de truchas en diversos ríos y lagos.

\section{Etapa 2: Gestión Estatal}

En 1930 llegaron a Chile desde Estados Unidos 114 mil ovas de salmón rojo y 225 mil de salmón coho, pero es probable que debido al desconocimiento de las condiciones ambientales y de circulación de corrientes marinas los intentos por afianzar cultivos fracasaran. Si bien la producción de la piscicultura Lautaro fue considerable entre 1928 y 1932, a partir de 1934 empezaron a disminuir las poblaciones y en 1937 desaparecieron definitivamente por causas no establecidas. A pesar de esta mala experiencia la piscicultura Lautaro siguió cultivando truchas, lo que permitió iniciar la exportación de ovas a Perú, Argentina, Colombia, Ecuador, Bolivia e incluso a las islas Falkland, situación que se mantuvo hasta 1957. Años antes (1953), se construyó la tercera estación de cultivo de salmónidos en el país, en la localidad de Polcura, donde se trabajó con truchas arco iris y café.

En esa época, el estado contó con la cooperación de expertos norteamericanos, como el Dr. John Dewitt y Harry Gibson, quienes asesoraban las investigaciones de funcionarios de la División de Pesca y Caza del Servicio Agrícola y Ganadero, SAG, dependiente del Ministerio de Agricultura - que más tarde derivaría en lo que hoy es el Servicio Nacional de Pesca (Sernapesca) acerca de los rasgos biológicos y geográficos de la zona. Este organismo, con asistencia de algunas universidades y miembros del Cuerpo de Paz de Estados Unidos, quienes realizaban docencia e investigación en el Instituto Tecnológico de Puerto Montt -dependiente de 
la Universidad Técnica del Estado y que transferían la tecnología norteamericana de cultivo de truchas y salmones- financiaba y operaba los proyectos estatales para introducir y repoblar con truchas los cursos de agua del país mediante la construcción de pisciculturas, como la del río Lautaro, instalaciones mitilicultoras (para cultivo de mitilílidos, como choritos) y ostriculturas (para cultivo de ostras). En la misma época, el gobierno de turno comenzó a apoyar la iniciativa del cultivo abierto u ocean ranching, en el cual, a diferencia de lo que sucede con el cultivo en cautiverio, los alevines son criados en estanques y luego liberados en el mar para que, siguiendo su ciclo natural, remonten los ríos y regresen a su lugar de origen a la hora de desovar, donde son capturados. El Estado también dedicó esfuerzos a desarrollar las capacidades humanas relativas a la pesca y al sector acuicultor con la creación de las carreras de Biología Marina, Oceanografía e Ingeniería en Pesca y estableció los organismos dedicados a la investigación en acuicultura (como el Instituto de Fomento Pesquero, IFOP).

Fundado a mediados de la década del 60 con el apoyo del gobierno chileno y de la FAO (Food and Agriculture Organization of de United Nations) desde sus inicios fue una pieza clave en el desarrollo y consolidación de la acuicultura debido a que trajo al país a los primeros expertos extranjeros en el tema, quienes generaron las tecnologías para cultivar mitílidos en el sur de Chile. De hecho, a fines de 1964 los funcionarios del IFOP Silvia Soto, Héctor Trujillo y José Raúl Cañon recorrieron por cerca de tres meses la zona comprendida entre Puerto Montt y Punta Arenas, por tierra, aire y mar, evaluando la calidad de las aguas de ríos y lagos para sembrar semillas de choritos. En ese entonces, su conclusión fue que existían lagos y ríos aptos para la actividad.

Aunque el país estaba en condiciones de cultivar salmones en aguas dulces gracias al conocimiento adquirido sobre técnicas de incubación y alevinaje de truchas, no existía ningún antecedente sobre la fase marina del cultivo de los primeros. Todos los proyectos demostraban factibilidad técnica, pero no había resultados en la factibilidad económica y sus mercados permanecían desconocidos.

En este contexto comenzaron a trabajar quienes hoy son catalogados como los pioneros de la acuicultura en Chile. Pablo Aguilera, en ese entonces funcionario del SAG (Servicio Agrícola y Ganadero) en Coyhaique, realizó en 1968 el primer traslado aéreo de ovas de trucha desde la piscicultura Lautaro para sembrarlas en los ríos y lagos aledaños a Coyhaique. En tanto, Andrés Couve Rioseco -Director de la División de Pesca y Caza del SAG entre 1967 y 1970 (y hoy empresario pesquero)- gracias a un encuentro con el Dr. Ernest Salo, profesor de School of Fisheries de la Universidad de Washington en Seattle (Estados Unidos) firmó un convenio entre dicha casa de estudios norteamericana y la entidad que dirigía para seleccionar los ríos adecuados e introducir el salmón coho y Chinook entre Valdivia y Puerto Montt. El Dr. Salo tenía experiencia en salmonicultura puesto que había instalado en forma experimental algunas balsas-jaulas (estructuras para la crianza de peces en cautiverio en grandes cuerpos de agua) en su país para cultivar salmones. Ambos recorrieron el sur buscando lugares propicios y finalmente diseminaron ovas de salmón coho, rosado y rey en el río Chirri y en el estero La Zorra, el mismo que en 1968 recibió la siembra de 12 mil alevines de salmón coho donados por el Dr. John Dewitt, de Humboldt State College. El resultado no fue el esperado, puesto que hubo $80 \%$ de mortalidad.

Entre 1969 y 1970, la Comisión Pesquera del Estado de Oregón, Estados Unidos, regaló otra partida de alevines que también se sembraron en dicho estero, mientras el río Chirri recibió 100 mil alevines de salmón rey. La iniciativa tropezó con problemas políticos y sociales; el SAG no pudo seguir atendiendo el programa y no fue posible recabar información de retornos de peces, pero el proyecto marcó un gran progreso en el conocimiento de técnicas de cultivo, especialmente en temas relativos a dietas, control de enfermedades y transporte de peces. 
También bajo la administración de Andrés Couve en la división de Pesca y Caza del SAG se inició la crianza experimental de salmones en balsas-jaulas en Chinquihue, y en 1971 se terminó de construir la piscicultura de Pullinque (cerca del lago Panguipulli) dedicada al cultivo de truchas y que actualmente está en manos de la Municipalidad de Panguipulli.

Hubo otros intentos por introducir truchas y salmones en Chile, por ejemplo el proyecto de la Poza en la piscicultura de Río Blanco, en Los Andes, llevado a cabo por la experta en limnología Irma Vila -quien en 1964 trabajaba en la División de Pesca y Caza del SAG- y por Igor Solar.

En este contexto, en 1969 se formalizó el interés por introducir el salmón coho en las XI y XII regiones gracias al "Programa de Introducción de Salmón coho en Chile", que formaba parte de un convenio entre los gobiernos de nuestro país y de Japón, con la participación de la División de Pesca y Caza del SAG y de expertos de la Agencia Internacional de Cooperación Japonesa (JICA) y de la Asociación de Pesquerías de Japón.

Fruto de este acuerdo, en 1972 se inició en el río Claro, Aysén, la incubación y alevinaje de 150 mil ovas de salmón sakura en la piscicultura provisoria que, finalmente, sería arrastrada por la crecida de ese curso de agua. Dos años más tarde comenzó a construirse la piscicultura Dr. Yoshikazu Shiraishi, donde se incubaron un millón de ovas de salmón perro. Ello ocurrió en gran parte gracias a la gestión de Pablo Aguilera, quien en dos años antes, en 1970, había estado en Japón becado por un año y ya de vuelta en el país había ingresado a trabajar en el marco del convenio JICA con ideas nuevas sobre técnicas de cultivo y convencido de que sería factible practicar en Chile la técnica del raching utilizada en Japón para liberar Smolts (salmones juveniles al momento de iniciar su adaptación el medio marino). Sernapesca contrató colaboradores para sembrar truchas en el margen sur del lago General Carrera, en todos los lagos de la cuenca de Baker hasta el lago O'Higgins y en las lagunas al sur de Coyhaique hasta Puyuhuapi por el norte, cubriendo el $100 \%$ de la zona continental de Aysén.

Aviones particulares, helicópteros de la Armada y del Ejercito realizaban prospecciones aéreas de los mejores cursos de agua mientras hombres a caballo hacían recorridos de hasta seis horas. Los retornos no alcanzaron el $1 \%$ y no tuvieron relevancia económica.

\section{Etapa 3: Gestión industrial, apertura a mercados internacionales}

Una de las primeras iniciativas privadas que daría un giro radical a la salmonicultura en Chile se desarrolló en 1974 con el inicio del cultivo de trucha arco iris con fines netamente comerciales, tanto para consumo nacional como de exportación, gracias a un préstamo de US $\$ 100$ mil otorgado por la Corporación de Fomento de la Producción (CORFO) a la empresa Sociedad de Pesquerías Piscicultura Lago Llanquihue Ltda., (cuyas instalaciones fueron compradas posteriormente por Salmones Llanquihue S.A.).

Creada en 1975 por Alfredo Valenzuela y Alfonso Muena, la empresa trabajaba en el esquema tradicional del cultivo en estanques de concreto armado de gran capacidad en la superficie del agua, mientras en estanques de tierra se mantenían a los ejemplares reproductores. Cuando se comprobó que las ovas nacionales no daban buenos resultados, la empresa comenzó a importarlas. En 1978 se realizó la primera exportación a Francia con una excelente acogida, y pronto los envíos alcanzaron otros países de Europa y, en el mismo año, penetraron el mercado norteamericano.

En 1976, la empresa Union Carbide, dueña de Domsea Farms en Washington, Estados Unidos, dio un nuevo impulso al Raching al manifestar interés por el cultivo abierto del salmón en Chile 
con fines comerciales. Su filial chilena, Union Carbide Comercial Chile Ltda., y la División de Protección Pesquera del SAG -que reemplazó a la División de Pesca y Caza del mismo servicio- recomendaron la zona de Chiloé, por su similitud con las condiciones geográficas en que operaba su empresa en Estados Unidos. Finalmente se eligió el lago Popetán, en Chiloé, donde se construyó un muelle y se instalaron dos pequeñas jaulas para alevines. Ese mismo año llegaron 500 mil ovas de salmón coho, pero el 90\% murió por una inesperada alza de temperatura. Domsea Farms envió desde Estados Unidos una partida adicional 200 mil ovas que se sembraron en Curaco de Vélez, de Chiloé, actualmente reconocida en todo el mundo por sus excelentes aptitudes para cultivar salmones.

En 1977 se inició un cultivo de circuito abierto y se inauguró una piscicultura donde, hasta hoy, se desarrolla esa actividad en estanques de tierra para las fases de alevinaje. En 1978 se liberaron más de 170 mil alevines de salmón Chinook en Curaco de Vélez. Los retornos no alcanzaron sin embargo el $1 \%$.

En 1979 la empresa pasó a ser propiedad de Domsea Pesquera Chile Ltda., que siguió liberando especímenes en Curaco de Vélez. Entre 1978 y 1982 se liberaron alrededor de 600 mil alevines de salmón coho y 400 mil de salón Chinook, pero ese mismo año, Union Carbide se marginó del negocio de los salmones y, tanto la piscicultura como Domsea Farms y Domsea Pesquera Chile Ltda., fueron adquiridas por Campbell Soup, empresa que no siguió trabajando en el país. Al igual que en Aysén el Ranching tampoco tuvo éxito en Curaco de Vélez.

Regresando algunos años atrás, otro hito fue la construcción en 1976 de la piscicultura de río Sur, en la Región de Los Lagos, destinada al cultivo de truchas y propiedad del Instituto Tecnológico de Puerto Montt. La iniciativa se concretó con aportes del Club de Pesca y Caza de la zona y del amante de la pesca deportiva Erico Neumann. De esa piscicultura surgió la primera población de salmones del lago Llanquihue, luego que en 1976 el administrador de la piscicultura, Fernando Berroeta, enviara desde Estados Unidos 100 mil ovas de salmón coho. De ellas nacieron 60 mil alevines, pero como las defensas y prevenciones para evitar escapes estaban ideadas para truchas y no para salmones, 30 mil ejemplares escaparon hacia el mar por el Río Sur, afluente del Río Pescado, que a su vez es tributario del lago Llanquihue. Sin embargo, un año y medio más tarde los salmones comenzaron a retornar hacia el lugar del que habían partido.

El Estado siguió aportando al desarrollo de la salmonicultura, y en 1978, creó la Subsecretaría de Pesca y el Servicio Nacional de Pesca, Sernapesca, el Ministerio de Economía, Fomento y Reconstrucción, en reemplazo de la División de Protección Pesquera del SAG. El convenio JICA continuó así bajo la tuición de la Subsecretaría de Pesca y de Sernapesca como organismo encargado de la parte técnica. Se fijaron también un marco legal específico para regular la actividad, así como fondos concursables e instrumentos para financiar proyectos de investigación y de transferencia tecnológica para la acuicultura.

De esta manera, a fines de los 70 , se hacía cada vez más evidente que el interés demostrado por los Estados Unidos y Noruega por cultivar salmones era replicable en Chile. Las personas que ya habían trabajado en los intentos de poblar de salmones los cursos de agua en el país estaban capacitadas para empezar a trabajar la salmonicultura con fines comerciales, lo que constituyó la revolución más importante que ha tenido la acuicultura nacional. Demostración de lo anterior fue el surgimiento en 1979 de la empresa Nichiro Chile Ltda., que marcó el primer esfuerzo comercial formal por cultivar salmones para el mercado japonés en balsas-jaulas en mar con ovas importadas de Estados Unidos. Nichiro Chile utilizó desde el principio jaulas de metal, y la crianza de los primeros smolts de salmón coho fue realizada en la Sociedad de Pesquerías Piscicultura Lago Llanquihue Ltda., a partir de 1980 comenzó la incubación y 
alevinaje en la piscicultura de Teobaldo Stange, en Chamiza (Puerto Montt) construida artesanalmente entre 1969 y 1972 con materiales reciclados de faenas agrícolas, como estanques de metal para la leche y barriles de madera donde se criaban las truchas para luego trasladarlas y sembraras en dos lagunas del mismo predio agrícola.

Los japoneses decidieron desarrollar la piscicultura familiar con capital y conocimientos técnicos para engordar smolts en ellos, y en enero de 1979 se recibieron las primeras 200 mil ovas de salmón coho. Los resultados fueron tan buenos que en 1980 se trasladaron los alevines a nuevas piscinas en el río Correntoso, X Región, hoy Región de Los Lagos. Por disposiciones estipuladas en la autorización inicial para el funcionamiento de la empresa, entre 1980 y 1982 Nichiro estuvo obligada a liberar un $10 \%$ de su producción de smolts para establecer un ranching, en 1980 liberó alrededor de 17 mil en el río Correntoso, los que se perdieron debido a que no sabían alimentarse solos y migraron todos a Angelmó, donde fueron capturados con anzuelos.

En 1979 se había concretado otra iniciativa privada en la Sociedad Pesquera Mytilus Ltda., propiedad de Pesquera Coloso y de la Industria Chilena de Alambre (Inchalam), que más tarde se convertiría en Pesquera Mares Australes Ltda. y actualmente es parte de Marine Harvest Chile S.A. del grupo Nutreco. La Sociedad Pesquera Mytilus Ltda., fue la primera en abordar el negocio de la salmonicultura en forma masiva y estructurada, con un nivel de inversiones mucho mayor que el del resto de sus competidoras gracias a una mejor tecnología aplicada y a un concepto altamente innovador en la crianza de salmones. Además, fue la primera en detectar la enorme ventaja que tenía Chile para practicar salmonicultura en lagos; comenzó con cultivos en zonas lacustres cuando todas las demás compañías lo hacían aún en piscicultura. En 1979 importó 110 mil ovas de salmón coho y, pese a que una floración de algas específicamente marea roja- produjo una fuerte mortandad en 1983 en las zonas de Maullín, Angelmó, isla Los Curas y Calbuco en la Región de Los Lagos, más adelante, tuvo que enfrentar otra fuerte pérdida por el uso de harina de pescado contaminada, fue la primera empresa en usar balsas-jaulas de metal galvanizado provenientes de Noruega y en lograr índices interesantes de exportaciones de salmón a Estados Unidos.

En este contexto, ¿Cómo opera el modo de apropiación territorial y bajo qué dinámicas económicas? En 1985 existían 36 centros de cultivo operando y la producción se elevó a más de 1.200 toneladas. Chile pasó a integrar el grupo de países productores salmónidos. Entre las empresas que surgieron en la época destaca Salmones Huillinco S.A. en 1987, que nació con el objetivo de iniciar la producción del salmón del Atlántico en el país. El 25\% del capital estaba en manos de la Fundación Chile y el resto correspondía a inversionistas nacionales y extranjeros. La producción y comercialización de esta empresa alcanzó alrededor de 600 mil alevines al año de salmón del Atlántico, para lo que se dispuso una piscicultura en el lago Huillinco, Isla de Chiloé.

En tanto, Finamar se dedicó en 1987 a la producción y comercialización de salmón ahumado para los mercados nacional y extranjero. Para ello, construyó en la comuna de Pudahuel, Región Metropolitana, una planta industrial no contaminante, dotada de tecnología y equipamiento de punta. El año siguiente, y orientada hacia la investigación de técnicas de crianza y reproducción de salmónidos, apareció bajo el alero de Fundación Chile la empresa Salmotec, que instaló sus plantas en Chiloé (Piscicultura Llau Llao) y Magallanes (Piscicultura Río Prat).

En la misma época apareció Salmones Pacífico Sur S.A., que partió de la mano de los hermanos Humberto y Claudio Fisher con un centro para engorda de salmones en el canal 
Abtao, en Chayahué, Región de Los Lagos, y que en 1995 se asoció con Salmones Huillinco, con la cual más tarde formaría la subsidiaria Gentec, dedicada a programas genéticos.

En 1997, Salmones Pacífico Sur adquirió el 50\% de la planta de procesos Salmopack y el 50\% de la empresa Antares, filial de AquaChile, con la que se fusionó totalmente en 1998. Otras compañías son Pacific Star, que se instaló en Quellón, Chiloé, bajo el nombre de Salmones del Sur y Pesquera Friosur, ligada al grupo Derco de la Familia Del Río, que surgió en la Región de la Araucanía para dedicarse al cultivo del Salmón coho en Puerto Chacabuco. En 1991 Salmones Friosur se constituyó como una empresa independiente y se trasladó a la zona de Santa María del Mar, siendo la primera empresa de la XI Región en cultivar salmón del Atlántico con smolts de la compañía Chisal S.A. Además, fue pionera en los centros de cultivo de la costa al instalar uno en 1988 a cinco horas de Puerto Chacabuco, en un período en que éstos estaban muy cerca entre sí.

Mares Australes y Nichiro Chile seguían siendo las más grandes de la industria. Gracias a estas empresas y al aporte de Fundación Chile se abrieron los mercados de exportación, así como también gracias al trabajo de personas como René Acklin, quien estableció contactos en el extranjero y golpeó muchas puertas para dar a conocer el salmón chileno en el mundo. De igual modo Fundación Chile formó un equipo de trabajo multidisciplinario en áreas como patología de salmónidos y construcción de balsas, con la asesoría de técnicos extranjeros y chilenos como Vjekoslav Rafaelli y Ximena Rivas.

Antes, en 1987 finalizó el convenio JICA, habiéndose recibido de Japón un total aproximado de 40 millones de ovas, que significó la liberación de 26 millones de juveniles de salmón perro y constituyó el esfuerzo de trasplante de salmón de mayor envergadura y continuidad desarrollado en el hemisferio sur, aunque no logró el objetivo inicial de introducir esa especie como población silvestre; los niveles de retorno a Aysén fueron muy bajos y sólo se obtuvieron mejores resultados con retornantes maduros en Puertos Natales.

Cabe aquí destacar que un poco antes (1986) comenzó el auge de la industria salmonicultora. Los proyectos de factibilidad económica arrojaban cifras impresionantes en relación con las tasas internas de retorno y la producción superaba las 2.100 toneladas brutas de salmón coho y trucha. Se tramitaron autorizaciones para 26 nuevos centros de cultivo, cifra que se elevó a 56 en 1987, año en que se instalaron cerca de 117 centros. Los flujos netos actualizados eran estimulantes y los tiempos de recuperación del capital superaban las estimaciones de la mayoría de los productos de inversión emprendidos en el país. En este escenario, Fundación Chile dio inicio a un ciclo de seminarios internacionales sobre salmonicultura en el país, que continuaron en 1987 y 1988 con representantes de Noruega, Japón, Escocia y Estados Unidos, entre otros. También se embarcó en una serie de acciones tendientes a generar conciencia en diversos grupos de interés sobre la importancia de la salmonicultura y así impulsar y facilitar su desarrollo.

En un trabajo intenso y dedicado, tomó contacto con distintos sectores económicos y consiguió, por ejemplo, que se crearan seguros para la salmonicultura, ayudando a generar un ambiente que otorgó tranquilidad y confianza a productores y compradores. En resumen, comprometió a las autoridades y al país a impulsar una actividad que abría una interesante posibilidad de negocios. Al mismo tiempo, Salmones Antártica logró positivos avances en la evaluación biotécnica del cultivo del salmón coho a mar abierto. Las exportaciones de salmón fresco de la empresa generaron más de $\$ 1$ millón (una década después, el año 96, se realizó por primera vez una exportación de salmón fresco por vía aérea a Estados Unidos directamente desde Balmaceda). 
También en 1986 inició sus operaciones la empresa de Salmones Unimarc S.A. que, durante muchos años fue la mayor productora mundial de salmón coho, con centros de mar, pisciculturas y planta de proceso en Chiloé. Después se formaron compañías como Aguas Claras, que desde sus inicios ha estado orientada a productos de alto valor agregado; Ventisqueros, que se instaló en el área del río Negro con muchas dificultades debido a la falta de caminos, puentes y energía eléctrica, logrando operar dos pisciculturas y dos centros de mar; Invertec Pesquera Mar Chiloé, en 1988, que aunque en sus inicios sólo produjo salmón coho, rápidamente se diversificó a otros salmónidos y a ostiones; Cultivos Marinos Chiloé, con instalaciones de procesamiento y fabricas de alimento en Ancud y centros de mar en islas Butachauques y en Puerto Cisnes: y pesquera Los Fiordos, que en 1989 fue pionera con centros de mar y operaciones en el área de Puerto Cisnes, y que en 2002 inauguró la planta de procesamiento más moderna de Chile en Quellón, Chiloé.

En el ámbito empresarial, Multiexport S.A. instaló en Puerto Montt la empresa Alimentos Multiexport S.A., dedicada a comprar materias primas a los pescadores artesanales y a la flota pesquera industrial para procesarlas y exportarlas como productos frescos, congelados y en conserva. En 1988 comenzó a ofrecer servicios a la industria salmonicultora, transformándose en la primera compañía que trajo a Chile desde Europa máquinas para procesar salmón. En 1989 estableció un centro de cultivos de salmones en su concesión en Dalcahue, Chiloé, llamado Salmones Multiexport Ltda. en 1995, la empresa se asoció a Chisal S.A. -propiedad de Corpora- y constituyeron la empresa Prosmolt S.A. para producir y comercializar ovas, alevines, smolts de salmón Atlántico y trucha arco iris. En 1998, Salmones Multiexport absorbió a Alimentos Multiexport S.A. y, un año más tarde, adquirió la totalidad de los activos productivos de Chisal S.A., para después comprar todas las acciones de Prosmolt S.A. y la Piscicultura del río Bueno S.A.

Por su parte la empresa conservera Robinson Crusoe, que comenzó exportando conservas a oriente y a Europa a partir de la pesca extractiva y también se inició en el negocio salmonicultor. En tanto, en 1987 se formó la empresa Patagonia Salmon Farming en Ilque, cerca de Puerto Montt, de propiedad de la familia Kossman.

Empresas dedicadas a otras actividades también incursionaron en la producción de salmónidos, entre ellas, Pesquera Camanchaca S.A., fabricante y exportadora de harina y aceite de pescado, que instaló su división salmones en la región de Los Lagos. En tant, Pesquera Yadrán S.A. procesadora y exportadora de productos del mar, instalada en Quellón con su planta de procesamiento, incorporó el cultivo de salmones y truchas como una continuación natural de sus actividades en la zona de Chiloé.

Marine Harvest, con operaciones productivas en Chile, Noruega, Escocia, Irlanda, Canadá y Australia, es la principal productora y exportadora de salmónidos en el mundo y también en Chile, con el nombre de Marine Harvest Chile S.A. creada en 1986. En 1999 el grupo holandés Nutreco adquirió Marine Harvest, con todas sus instalaciones en el mundo, y se fusionó con la empresa que tenía en Chile, Mares Australes. Marine Harvest Chile S.A -que en el año 2002 compró la planta de proceso de Chisal S.A. en el sector de Chamiza (Puerto Montt)- produce salmón del Atlántico, salmón coho y trucha arco iris y abarca cultivo en agua dulce y mar, operaciones en plantas de proceso y comercialización e la Región de Los Lagos.

Las empresas chilenas productoras de salmónidos tienen un prestigio relativo a nivel mundial y varias de ellas han sido adquiridas por grandes compañías internacionales. Es el caso de Salmones y Alimentos Mainstream, creada por la familia empresarial Furman en 1988 para cultivar salmones en Chiloé y fabricar alimentos para peces en Coronel (Región del Bío Bío). La planta de alimentos para salmónidos fue adquirida por Ewos (empresa de origen noruego) en 
1995, mientras que en el área de salmones creció en manos de sus nuevos dueños "la familia Eblen" y otros socios, para convertirse en la primera empresa de salmones que se incorporó a la bolsa de comercio de Santiago. Ewos Chile fue adquirida en 2000 por Cermaq ASA, empresa noruega que también tiene operaciones en la salmonicultura de su país de origen, en Canadá y Escocia.

Otra compañía noruega que se instaló en Chile por medio de la compra de empresas nacionales es Fjord Seafood ASA, que en el 200 adquirió Salmoamérica, Cultivo de Salmones Linao y Salmones Tecmar. Incorporó estas empresas logrando reunir centros de mar, pisciculturas y plantas de procesamiento en grandes áreas de Chiloé y Puerto Montt, para así convertirse en el segundo mayor productor mundial de salmónidos, con instalaciones en 15 países. Finalmente, otra de las grandes compañías noruegas, Stolt Sea Farm, que poseía el $12,5 \%$ de Eicosal desde 1994 y el $100 \%$ de Ocean Horizons, de origen chileno desde el año 2000, decidió comprar la totalidad de la primera en 2001. Así, estas empresas pasaron a integrar Stolt Sea Farm Group, una compañía de acuicultura mundial que data desde 1972.

En tanto, Pesca Chile S.A., del grupo español Pescanova, dedicada a la pesca en el país desde 1983, decidió empezar a cultivar salmones en la Región de Aysén en el año 1991 para aprovechar su planta de procesamiento en Chacabuco. El mismo razonamiento la llevó a iniciar significativas operaciones de intervención y reapropiación en las zonas cercanas a Punta Arenas y Puertos Natales desde el 2001.

Una muestra de consolidación definitiva de la industria salmonicultora la constituye la creación, en 1986, de la Asociación de Productores de Salmón y Trucha de Chile A.G. -que en mayo del 2002 pasó a llamarse Asociación de la Industria del Salmón A.G., SalmónChile-, con 17 compañías dedicadas a la actividad. Impulsada por Fundación Chile, su objetivo fue generar un sello de calidad para producción y promoción del salmón chileno en los mercados mundiales mediante el establecimiento de requisitos mínimos que debían cumplir las plantas procesadoras como referencia para obtener una mercadería de óptima calidad y a los que debían adherir todos los miembros de la entidad. SalmonChile agrupa a los productores de salmónidos responsables del $83 \%$ de las exportaciones de esta industria en Chile.

Regresando al año 1987, cabe precisar que se registraron las primeras exportaciones de salmón del Atlántico hacia Estados Unidos. En forma bastante rudimentaria, lanchas chilotas trasladaban los ejemplares recién cosechados en cajas de madera con hielo desde las balsasjaulas hasta los muelles para llevarlos a las plantas procesadoras, donde se limpiaban y empacaban en cajas de poliestireno expandido o plumavit con hielo y se enviaban al aeropuerto. Allí se colocaban en contenedores y se enviaban en aviones de pasajeros a su destino.

En forma simultánea al aumento en el volumen de producción y a las exigencias del mercado, la industria salmonicultora nacional comenzó a vivir un explosivo desarrollo. Empezó a evolucionar la metodología de cosecha y se registraron cambios importantes en el manejo y control experimental de peces, la cadena de frío y la mecanización de los sistemas de extracción. Junto a lo anterior, la empresa Marine Harvest hizo debutar los Bins, recipientes de plástico con aislación térmica.

De forma también paralela, Fundación Chile, creó Salmones Huillinco, una de las primeras en trabajar con smolts de salmón del Atlántico en Chile, y que -en el marco de la labor de Fundación Chile de iniciar actividades productivas innovadoras- licitó públicamente en 1988 Salmones Antártica, que fue adjudicada a la empresa japonesa Nippon Suisan. Salmones Antártica dejó huella como la impulsora en el traspaso de desarrollo tecnológico en el cultivo de 
salmones; fue la primera empresa chilena que sobrepasó las mil toneladas de producción de salmón durante la temporada 1987-1988 y la única que mantuvo programas sostenidos de cultivo a mar abierto en Chiloé y Magallanes, en la piscicultura más austral del mundo.

En 1990, la salmonicultura comenzó a desarrollar reproducción en Chile y se obtuvieron las primeras ovas nacionales de salmón coho. Este hito se recuerda como el primer adelanto científico significativo chileno, el primer síntoma de emancipación de los mercados proveedores, lo que significó el despegue de la industria y un cambio importante en el ciclo productivo del salmón coho. Hatfield fue la empresa pionera en la producción de una cantidad importante de ovas de esa especie, pero en 1992 se marginó del negocio salmonicultor al vender los capitales que tenía comprometidos en la compañía, pasando a llamarse Ventisqueros S.A.

En 1995 se creó el Instituto Tecnológico del Salmón, Intesal, por iniciativa de la Asociación de productores del Salmón y Trucha de Chile A.G. -hoy SalmónChile-, con el objetivo de aumentar la eficiencia productiva de la industria y preocuparse de los aspectos sanitarios, medioambiental y de capacitación.

De la década de 90 datan las supuestas mejoras en los alimentos para salmones. A medida que los volúmenes de producción requeridos fueron creciendo, se hizo necesario contar con un proveedor profesional y especializado en el tema, pues tres años antes (1987) se optó por la tecnología de pelletización, produciéndose alimentos con alto contenido de proteínas y bajo aporte de lípidos. Luego, la incorporación de tecnología de extrusión permitió fabricar alimentos secos con crecientes contenidos de lípidos y un balance más eficiente entre éstos y las proteínas, hasta que el desarrollo de los aceitadores al vacío, a mediados de la década, permitió iniciar la fabricación de alimentos que contienen hasta el $40 \%$ de lípidos, un factor determinante para lograr un mayor crecimiento de los peces.

Agropellet y Surlim comenzaron a ofrecer alimento concentrado fabricados industrialmente y surgieron importantes empresas concebidas para producir alimentos para salmones; Alimentos Mainstream, Trow Chile S.A. (actualmente Skretting), que durante la década del 90 ofreció las dietas con mayores niveles de energía o lípidos del mercado.

Actualmente se siguen entregando pellets a los peces varias veces de forma manual, se cuenta con raciones diseñadas para cada balsa-jaula. También hay sistemas automáticos de alimentación que por medio de sensores y cámaras de video permiten identificar el momento en que los peces dejan de alimentarse para suspender el suministro y no desperdiciar alimento.

Junto con los cambios en los procesos de alimentación, la industria también modificó técnicas de cultivo. Actualmente, los peces se trasladan del agua hacia los equipos de selección con bombas y no solo con quechas (canastillo de mallas con mango que se usan para capturar peces); el conteo, selección y graduación se realiza por lo general en una sola operación, para disminuir la manipulación de los salmónidos.

En 1998 la industria vivió uno de sus momentos más complicados debido a la crisis asiática que hizo caer los precios en Japón- y a una sobreproducción a nivel mundial.

\section{Conclusiones}

Hemos dado cuenta de una sistematización -al modo de una breve historia de la acuicultura- de datos que nos permiten identificar procesos y actores relevantes de su evolución y desarrollo, como también, etapas y procesos. Al iniciarse de forma experimental a inicios del siglo decimonónico con objetivos muy distintos a su desarrollo final, la acuicultura representa un 
campo de exploración cuyo auge fundamental (aún no económico) se desarrolla a partir del impulso estatal décadas después generando las condiciones iniciales para su redefinición en el contexto empresarial, y con ello, la emergencia de intereses industriales.

Estas tres etapas identifican el acelerado proceso de reapropiación territorial de los espacios marítimo costeros del mar interior de Chiloé, sobre todo durante las últimos cincuenta años, donde los avances científicos en materia acuícola permitieron reinventar los procesos técnicos y fines del rubro. La incorporación de conglomerados económicos y la compleja red de compra venta desarrollados entre ellos durante el inicio del presente milenio contribuyó a la construcción de un nuevo concepto de la acuicultura el cual permeo en el desarrollo de políticas públicas que benefician directamente a la industria.

El punto de partida de la acuicultura en Chiloé refiere al año1976 cuando la empresa Union Carbide, dueña de Domsea Farms en Washington, Estados Unidos, dio un nuevo impulso al Raching al manifestar interés por el cultivo abierto del salmón en Chile con fines comerciales, pues, su filial chilena -Union Carbide Comercial Chile Ltda., y la División de Protección Pesquera del SAG- recomendaron la zona de Chiloé, por su similitud con las condiciones geográficas en que operaba su empresa en Estados Unidos. Desde entonces hasta el día de hoy, la Acuicultura -como modelo económico- se ha instalado en el territorio a partir de un discurso complejo que imbrica subjetivas confianzas en el conocimiento científico aplicado y que han repercutido de diversas formas, entre las más importantes, crisis socioecológicas que han generado un daño irreparable en el subsuelo marino del mar interior de Chiloé y la costa chilena.

Anclados en el discurso de la superación del hambre, la incorporación de bienes y servicios en territorios locales y la generación de empleos, la Acuicultura-Salmonicultura ha transformado los territorios locales en términos identitarios, culturales, económicos y urbanos.

En términos identitarios, los actores de los territorios locales se han desplazado desde los oficios tradicionales (con prácticas tradicionales rurales-costeras) hacia una nueva configuración de empleabilidad que prometía mejoras en la calidad de vida, pero que repercutieron en importantes movilizaciones sociales que evidenciaron las carentes condiciones laborales, trabajos de sueldos inferiores al mínimo y basados en horarios laborales de hasta 12 horas.

En términos culturales, la incorporación de un nuevo discurso anclado en la razón científica y económica desplazó las tradiciones locales convirtiéndolas -desde la perspectiva local- en fetiches turísticos, y con ello, una redefinición de sus prácticas cotidianas, sus paisajes, sus rutas, entre otros, proceso que expresa una disputa de sentidos aún en desarrollo y que se sitúa en un estadio de colonización de casi la totalidad de la isla, proceso amparado por los gobiernos sub-nacionales y locales (empleabilidad por sobre sustentabilidad de la biodiversidad). En términos económicos, el proceso de apropiación territorial evidencia el conjunto de garantías y facilidades que permitieron el desarrollo y auge de la industria, la inserción de lógicas y capitales extranjeros, y a su vez, el conjunto de normativas que desde Sernapesca e IFOP fueron legitimadas e instituidas fueron sancionadas (como significación válida y colectiva) sin un diseño de participación territorial, sin claridad del concepto de desarrollo propuesto y a partir de un diálogo asimétrico entre lo empresarial, estatal y local.

Este proceso implicó significativos cambios en el modo de pensar de los habitantes del borde costero, incluidos pescadores artesanales que cambiaron sus labores, y para quienes permanecieron debieron readaptarse a los nuevos criterios legales y jurídicos amparados en las lógicas del mercado y los principios de oferta y demanda, relaciones que cambiaron el paisaje 
urbano y contribuyeron a la urbanización de sus territorios sin quedar claro si ello significó un beneficio social o un problema ecológico hoy difícil de reparar.

Actualmente, la puga de sentidos y significaciones entre la empresa, el Estado y los habitantes es un proceso en desarrollo que trasciende el territorio local e incorpora nuevos actores institucionales y ha implicado una discusión política para redefinir la ocupación del mar interior de Chiloé, sus recursos, el acceso a éstos y las lógicas de desarrollo que han cosificado al modo de indicadores el territorio local.

\section{Referencias}

Amtmann, M., Fecci, E. y Gómez, S. (2004). Competencias laborales de la industria salmonera y mano de obra rural en la comuna de Dalcahue, Provincia de Chiloé. Estudio de caso. (Tesis de Magíster). Magíster en Desarrollo Rural, Universidad Austral de Chile.

Díaz, E. (2003). Transnacionalización de la industria salmonera. Aspectos socio-laborales de un proceso en curso. Santiago de Chile: Oxfam y Exagrama Consultoras.

Donoso, W. (2006). Salmonicultura: Pilar de crecimiento del sector Pesca y Acuicultura. FitchRatings. Reporte espacial.

García Allut, A. (2004). Boletín 44 del Instituto Andaluz del Patrimonio Histórico, ed. por la Junta de Andalucía un artículo sobre la patrimonialización del conocimiento ecológico tradicional en la pesca artesanal.

Martínez, S. (2003). Historia de la acuicultura. Santiago de Chile: TechnoPress S.A. Editorial de AquaNoticias. $1^{\circ}$ ED. Directorio de Acuicultura y Pesca de Chile SalmónChile.

Olguín, C. (1971). Instituciones políticas y administrativas de Chiloé en el siglo XVIII. Santiago de Chile: Editorial jurídica de Chile. Publicaciones del Seminario de Historia y Filosofía del Derecho de la Facultad de Ciencias Jurídicas y Sociales de la Universidad de Chile. Estudios de Derecho Indiano.

Ramírez, A. (2001). Modernización de la gestión pública. El caso chileno (1994-2000). Estudio de caso $n .{ }^{\circ} 58$. Magíster en políticas públicas. Universidad de Chile.

Ramírez, E. Modrego, F. Macé, J. C. y Yáñez, R. (2009). Caracterización de los actores de Chiloé Central. Documento de Trabajo n. ${ }^{\circ} 55$. Programa Dinámicas Territoriales Rurales. Santiago de Chile: Rimisp.

Selada, S. \& Park, J., (2013). Análisis crítico de la Ley Lafkenche (n. $\left.{ }^{\circ} 20.249\right)$. El complejo contexto ideológico, jurídico, administrativo y social que dificulta su aplicación. Universum, 28 (1).

Skewes, J., Álvarez, R. \& Navarro, M. (2012). Usos consuetudinarios, conflictos actuales y conservación en el borde costero de Chiloé insular. Magallania, 40 (1), 109-125. 
